\title{
A Novel Method of Fingerprint Classification Using Image Parameters on ANFIS
}

\author{
B R Deva Vikram and Rajesh Kumar, Senior Member, IAENG
}

\begin{abstract}
Numerous algorithms have been developed to improve the speed of fingerprint recognition and this assumes prime importance when dealing with a large database of fingerprint images. In this paper, we propose a novel technique of using the image parameters like mean, median, variance, standard deviation and root mean square value to train an adaptive network based fuzzy inference system (ANFIS) to classify the test image into one of the six popular categories of fingerprints namely arch, tented arch, left loop, right loop, whorl and twin loop. This classification allows us to proceed with the actual matching algorithm only with the images which fall in that particular category of ridge structure thereby saving time.
\end{abstract}

Index Terms - fingerprint classification, parameters, ANFIS, weights

\section{INTRODUCTION}

Fingerprint based identification is a popular and reliable biometric technique for personal identification. The property of these patterns, where they retain their uniqueness during the entire lifetime makes it a successful means of identification. The formation of this graphical flow like ridges on the human finger depends on the initial conditions of the embryonic mesoderm from which they develop [1].

The fingerprint is classified into a number of categories depending on the ridge flow pattern in its central region. One school of classification of ridge flow pattern is in to Wirbel and Lasso class [2]. The more popular method of classifying the fingerprint image is into arch, tented, left loop, right loop, whorl and twin loop. The classification of the fingerprint image is carried out before the actual matching, so that the number of images that have to be matched with the test image is reduced by one-sixth.

The different approaches used for the classification are grouped as: syntactic, singularities based, structural based and the neural network based [3]. The methods depending on the singular points, which are defined as the region where the orientation field is discontinuous, are quite popular. Representations based on principal component analysis (PCA) [4], self organizing map (SOM) [5] and gabor filters [6] are used. Some of the recent methods include the use of machine learning approaches like support vector machines

Manuscript received May 12, 2009.

Rajesh Kumar is with the Electrical Engineering Department, Malaviya National Institute of Technology, Jaipur, 302017, INDIA (corresponding author phone: 91-9413301134; fax: 91-141-2529029)

B R Deva Vikram was with MNIT, Jaipur, 302017, INDIA. He is now a graduate student in the Department of Electrical Engineering in Texas A\&M University USA.
(SVM), recursive neural network (RNN) [7]. The combination of hidden markov models (HMM), decision trees and PCASYS to improve the classification performance is also popular [8].

In all these conventional methods, the fingerprint images are firstly subjected to pre-processing and then the features are extracted which are then used for classifying the image into a particular category. These methods are found to be extremely cumbersome and time-consuming in it, thereby making it difficult to achieve our mission of reducing the time. In this paper, we propose a novel method for the classification of images where, we directly use the fingerprint image without any processing for extracting a few parameters namely the mean, median, variance, standard deviation and root mean square value from the image. These parameters are then used on an adaptive network based fuzzy inference system which is capable of classifying a test image into one of the six groups. This method is proposed as it uses the comprehensive data of the fingerprint and hence the chances of failure to recognize the authentic fingerprints are very remote.

The rest of the paper is organized as follows: Section II describes the image enhancement techniques along with the binarization techniques. Section III explains the parameter extraction and its authentication method. Section IV introduces the concept of ANFIS and its algorithm. The results are presented in section $\mathrm{V}$ and the concluding remarks are presented in section VI.

\section{IMAGE ENHANCEMENT:}

The quality of the fingerprint ridge structure is very important as they possess the necessary information for the extraction of minutiae points. Ideally the ridges and valleys should alternate with a clear demarcation and flow in a locally constant direction. Due to a number of factors, the obtained fingerprints may not have well defined ridge/valley structures and might contain a lot of disturbance in the image [1]. So the fingerprint image is first enhanced before further processing and comprises of the following steps (i) Segmentation (ii) Normalization (iii) Orientation estimation (iv)Ridge frequency estimation (v)Gabor filtering (vi) Binarization [11].

\section{A. Segmentation}

Segmentation is the process of distinguishing the background region from the foreground regions. The foreground region is referred to the area which comprises of the ridges and the valleys of the fingerprint. The background region corresponds to the area outside the boundaries of the fingerprint and is not in the area of interest. This is done in 
order to completely alienate the background area from interfering with the process of minutiae extraction and hence avoid the detection of false minutiae. It is observed that the background regions have a low gray-scale variance value as compared to the foreground region. So a method based on variance thresh holding is used for segmentation [5]. So if the variance is less than a certain value, then it is assigned to belong to the background region otherwise it is said to belong to the foreground. In this method the image is divided into a number of small blocks of size $\left(n^{*} n\right)$ and their gray-scale variance is defined as

$$
V(k)=\frac{1}{n^{2}} \sum_{i=0}^{n-1} \sum_{j=0}^{n-1}(I(i, j)-M(k))^{2}
$$

where $V(k)$ is the variance and $M(k)$ is the mean gray-level value of the block $k . I(i, j)$ is the pixel value at the pixel $(i, j)$.

\section{B. Normalization}

Normalization is done in order to standardize the dynamic variation in the gray-level values so that it lies within a desired range of value. This is done in order to facilitate the subsequent enhancement process.

$$
\begin{gathered}
N(i, j)=M_{o}+\sqrt{\frac{V_{o}(I(i, j)-M)^{2}}{V}} \quad \text { if } I(i, j)>M \\
M_{o}-\sqrt{\frac{V_{o}(I(i, j)-M)^{2}}{V}} \text { otherwise }
\end{gathered}
$$

where ' $N(i, j)$ ' is the normalized gray-level value and $I(i, j)$ is the gray-level value at pixel $(i, j)$. ' $M$ ' and ' $V$ ' are the estimated mean and variance of $I(i, j)$ respectively and ' $M_{o}$, and ' $V_{o}$ ' are the desired mean and variance values respectively.

\section{Orientation Estimation}

The orientation field of a fingerprint defines the local orientation of the ridges and is necessary for the Gabor filtering stage to effectively enhance the image [6]. The orientation at the pixel $(i, j)$ is evaluated as follows. A block of size $\left(W^{*} W\right)$ is centered at the pixel $(i, j)$ in the normalized image. The gradient magnitudes in the $\mathrm{x}$ and $\mathrm{y}$ directions, $\partial_{x}(i, j)$ and $\partial_{y}(i, j)$ respectively are computed by applying the horizontal and the vertical Sobel operator respectively [6]. The local orientation $\theta$ for the block centered at the pixel $(i, j)$ is calculated by using the following equations.

$$
\begin{gathered}
O_{x}(i, j)=\sum_{u=i-W / 2}^{i+W / 2} \sum_{v=j-W / 2}^{j+W / 2} 2 \partial_{x}(u, v) \partial_{y}(u, v) \\
O_{y}(i, j)=\sum_{u=i-W / 2}^{i+W / 2} \sum^{j+W / 2} \partial_{x}{ }^{2}(u, v) \partial_{y}{ }^{2}(u, v) \\
\theta(i, j)=\frac{1}{2} \tan ^{-1} \frac{O_{x}(i, j)}{O_{Y}(i, j)}
\end{gathered}
$$

The orientation image is converted into a continuous vector field. ' $\phi_{x}$ ', ' $\phi_{y}$ ' being the $x$ and $y$ components of the vector field respectively. The Gaussian smoothing is then performed and the equations for the process are as under,

$$
\begin{aligned}
& \phi_{x}(i, j)=\cos (2 \theta(i, j)) \\
& \phi_{y}(i, j)=\sin (2 \theta(i, j))
\end{aligned}
$$

$$
\phi_{x}{ }^{\prime}(i, j)=\sum_{u=\frac{-q}{2}}^{\frac{q}{2}} \sum_{v=\frac{-q}{2}}^{\frac{q}{2}} G(u, v) \phi_{x}(i-u w, j-v w)
$$

(9)

$\phi_{y}{ }^{\prime}(i, j)=\sum_{u=\frac{-q}{2}}^{\frac{q}{2}} \sum_{v=\frac{-q}{2}}^{\frac{q}{2}} G(u, v) \phi_{y}(i-u w, j-v w)$

where ' $G$ ' is a Gaussian low pass filter of size $q^{*} q$ and the final smoothened orientation field ' $O$ ' at pixel $(i, j)$ is given by

$$
O(i, j)=\frac{1}{2} \tan ^{-1} \frac{\phi_{x}{ }^{\prime}(i, j)}{\phi_{y}{ }^{\prime}(i, j)}
$$

\section{Ridge Frequency Estimation}

Along with the orientation field, the ridge frequency is also required for the construction of Gabor filter. This represents the local frequency of the ridge in the fingerprint. The image is first divided into small blocks and then the gray-scale values of the pixels in each block along a direction orthogonal to the local ridge orientation is plotted against the number of the pixels along the direction [3]. So we obtain a sinusoidal wave where the inverse of the number of pixels between the local minima points give the ridge frequency $F(i$, $j$ ) for that block centered at the pixel $(i, j)$.

\section{E. Gabor Filtering}

The orientation and the ridge frequency information is used to design the even-symmetric Gabor filter. The frequency and the orientation selective properties of this filter allows the filter to be tuned to give the maximum response to ridges at a specific orientation and frequency [16]. An even symmetric Gabor filter is defined in the spatial domain as

$$
\begin{aligned}
G(x, y ; \theta, f) & =\exp \left\{-\frac{1}{2}\left[\frac{x_{\theta}{ }^{2}}{\sigma_{x}{ }^{2}}+\frac{y_{\theta}{ }^{2}}{\sigma_{y}{ }^{2}}\right]\right\} \cos \left(2 \pi f x_{\theta}\right), \\
x_{\theta} & =x \cos \theta+y \sin \theta \\
y_{\theta} & =-x \sin \theta+y \cos \theta
\end{aligned}
$$

where $\theta$ and $f$ refer to the orientation and the frequency of the Gabor filter, while $\sigma_{x}$ and $\sigma_{y}$ refer to the standard deviation of the Gaussian envelope. The Gabor filter is applied to the fingerprint image by spatially convolving the image with the filter [16]. The application of the Gabor filter to the image is performed as follows.

$$
E(i, j)=\sum_{u=\frac{-M_{x}}{2}}^{\frac{M_{X}}{2}} \sum_{v=\frac{M_{y}}{2}}^{\frac{M_{y}}{2}} G(u, v ; O(i, j), F(i, j)) N(i-u, j-v)
$$

(15)

where $E, O, F, N$ are the enhanced, orientation, ridge frequency and normalized fingerprint images respectively. $M_{x}, M_{y}$ being the width and height of the Gabor filter mask. The range of frequency to which the filter responds is determined by the parameters $\sigma_{x}$ and $\sigma_{y}$ and this is chosen to be a function of the ridge frequency.

$$
\begin{aligned}
& \sigma_{x}=k_{x} F(i, j) \\
& \sigma_{y}=k_{y} F(i, j)
\end{aligned}
$$

In order to enable the filter to accommodate the majority of 
the Gabor waveform information, the filter size is allowed to vary according to the bandwidth of the Gabor waveform.

$$
\begin{aligned}
& M_{x}=6 \sigma_{x} \\
& M_{y}=6 \sigma_{y}
\end{aligned}
$$

where $k_{x}$ and $k_{y}$ are constants for $\sigma_{x}$ and $\sigma_{y}$ respectively.

\section{F. Binarization}

Binarization is performed to convert the gray-scale image into a binary image having either zero or one as the pixel intensity value depending on whether the gray-scale pixel intensity value was above or below a certain threshold [13]. This will help in clearly differentiating between the ridges and the valleys and would hence greatly help in locating the minutiae points.

\section{PARAMETER EXTRACTION:}

The classification of the image is achieved by extracting a set of features from the image which would be sufficient for realizing the category to which the image would belong. The set of features could be singular points, minutiae features or detecting the kernel that best fits the flow-field of the given fingerprint [9].

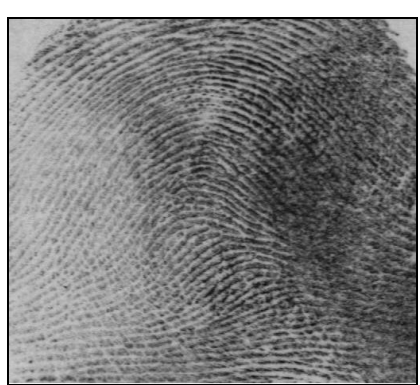

Fig 1. Arch type fingerprint

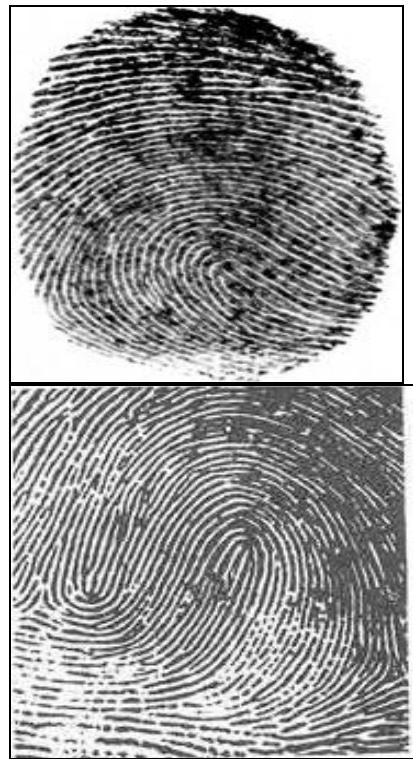

Fig 3. Left loop type fingerprint

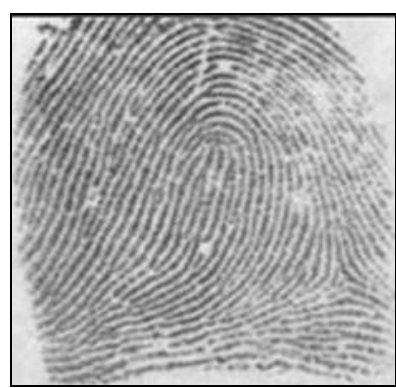

Fig 2. Right loop type fingerprint
Fig 4. Whorl type fingerprint

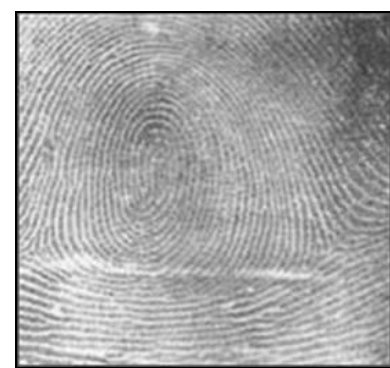

Fig 5. Twin loop type fingerprint

Generally, the task of finding a good feature is the most cumbersome part and in some cases the features to be extracted is also kept flexible and the system is designed to extract features which would best serve the purpose. In this paper, we use parameters like mean, median, variance, standard deviation and the root mean square value.

\section{A. Mean:}

The mean of the input image is defined as the average of the pixel intensity values of that image. In this method we firstly calculate the mean of the intensity values of the pixels along the rows for every column.

$$
\bar{x}(k)=\frac{1}{n} \sum_{i=1}^{n} x_{i}
$$

where ' $n$ ' refers to the number of elements, for which the mean is calculated, " $x_{i}$, refers to the individual elements, ، $\overline{x(k)}$, refers to the mean and ' $k$ ' refers to the image under consideration. This would result in a row vector with each element giving the mean of that particular column. The mean value of the elements of this row vector is calculated to give the final mean value.

\section{B. Median:}

The median of a finite list of numbers can be found by arranging all the observations from lowest value to highest value and picking the middle one. If there is an even number of observations, the median is not unique, so one often takes the mean of the two middle values. For example, if $a<b<c$, then the median of the list $\{a, b, c\}$ is $b$, and if $a<b<c<d$, then the median of the list $\{a, b, c, d\}$ is the mean of $b$ and $c$, i.e. it is $(b+c) / 2$. The median is calculated similar to the mean value for the image. The median is first calculated for all the elements which belong to the same column and then the median is calculated for this row vector.

\section{Variance:}

In this method, the variance of the image is calculated by initially finding it for the elements of the same column and then finally, the variance for this row vector is calculated. The variance for the set containing the pixel intensities value are given by

$$
v(k)=\frac{1}{n-1} \sum_{i=1}^{n}\left(x_{i}-\bar{x}\right)^{2}
$$

where ' $v(k)$ ' refers to the variance value.

\section{Standard deviation:}

The standard deviation of the image is the fourth parameter or the feature to be extracted from the image. This is also calculated as stated previously, by firstly getting the values 
along a single row and then calculating the final value of standard deviation of the image.

$$
s(k)=\left(\frac{1}{n-1} \sum_{i=1}^{n}\left(x_{i}-\bar{x}\right)^{2}\right)^{\frac{1}{2}}
$$

where ' $s(k)$ ' is the standard deviation. This is one of the important parameter used in the classification of the images.

\section{E. Root mean square value:}

The root mean square value of the image is calculated by using the expression

$$
r m s(k)=\sqrt{\frac{\sum_{i=1}^{m} I(i, j) * \overline{I(i, j)}}{m}}
$$

where ' $r m s(k)$ ' is the root mean square value, ' $I(i, j)$ ' is the intensity value of the pixel $(i, j), \overline{I(i, j)}$, refers to the complex conjugate of the pixel values and ' $m$ ' refers to the number of rows of the image. This is calculated in a similar manner to the previous parameter calculations with the first values extracted for all the elements of the same row and then the final value is extracted from this set.

\section{ADAPTIVE NETWORK BASED FUZZY INFERENCE SYSTEM}

The adaptive network based fuzzy inference system [10] is used for the classification of the fingerprint image into one of the six classes by using the image parameters extracted previously.

\section{A. Architecture}

This system can have a large number of inputs, but in order to make the explanation easier, let us consider a three input system. Suppose the rules are defined as

$$
\begin{aligned}
& \text { if } x \text { is } A_{1} \text { and } y \text { is } B_{1} \text { and } z \text { is } C_{1} \text { then } f_{1}=p_{1} x+q_{1} y+r_{1} z+s_{1} \\
& \text { if } x \text { is } A_{2} \text { and } y \text { is } B_{2} \text { and } z \text { is } C_{2} \text { then }
\end{aligned}
$$$$
f_{2}=p_{2} x+q_{2} y+r_{2} z+s_{2}
$$

The node functions in the same layer are of the same function family as described below:

\section{Stage 1: (layer2)}

$F_{i}^{1}$ is the membership function for $A_{i}$ and it defines the degree of membership of the input value ' $x$ ' with $A_{i}$.

$$
F_{i}^{1}=\mu_{A_{i}}(x)
$$

In this work, we have used the gaussian membership functions for fuzzifying the input values to a value in the range between 0 and 1 and they are defined as

$$
\mu_{A_{i}}(x)=a_{i} \cdot e^{\frac{-\left(x-b_{i}\right)^{2}}{2 c_{i}^{2}}}
$$

where $\left\{a_{i}, b_{i}, c_{i}\right\}$ is the parameter set. The values of the function changes according to the changes in these parameters.

\section{Stage 2: (layer3)}

In this stage, the incoming signals are processed to obtain the firing strength of a rule.

$$
w_{i}=\mu_{A_{i}}(x) \times \mu_{B_{i}}(y) \times \mu_{C_{i}}(z), \quad i=1 \ldots n
$$

where ' $n$ ' refers to the total number of rules. This value is used to obtain the ratio of the ' $i^{\text {th }}$ ' rule firing strength to the sum of all rules firing strength which are referred to as the normalized firing strengths.

$$
\overline{w_{i}}=\frac{w_{i}}{\sum_{k=1}^{n} w_{k}}
$$

\section{Stage 3: (layer4)}

The output functions are taken as linear in this work. In this stage we calculate the output values from the output functions.

$$
F_{i}^{4}=\overline{w_{i}} f_{i}=\overline{w_{i}}\left(p_{i} x+q_{i} y+r_{i} z+s_{i}\right)
$$

where $\left\{p_{i}, q_{i}, r_{i}, s_{i}\right\}$ are the parameters and are referred to as the consequent parameters.

\section{Stage 4: (layer5)}

The final output values are calculated by obtaining the sum of all the values obtained from the previous step.

$$
\text { overall-output }=\sum_{i} F_{i}^{4}
$$

In this work, we use the backpropogation learning algorithm and the gaussian membership function for fuzzifying the input variables.

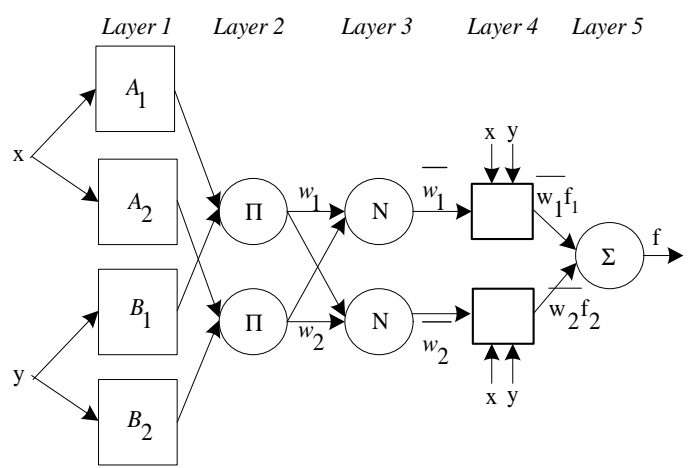

Fig. 6. ANFIS architecture of 2-input Sugeno fuzzy model with 2 rules.

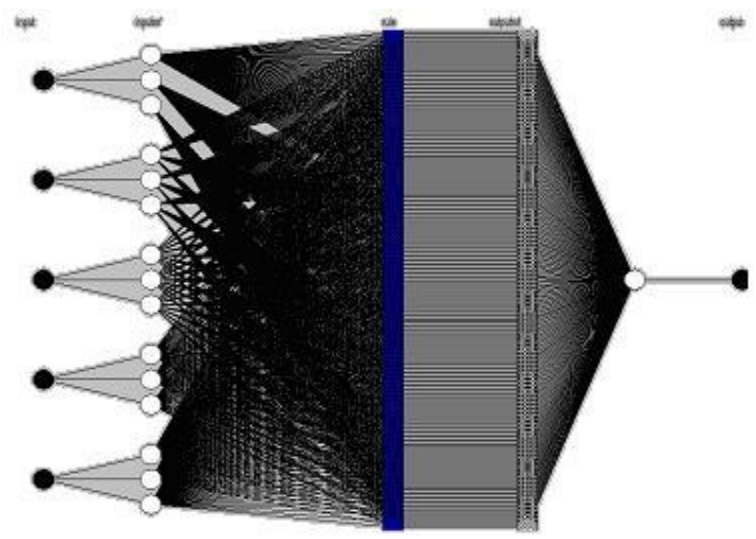

Fig 7: Structure of ANFIS with five inputs for fingerprint classification

\section{B. Backpropogation training algorithm:}

The training algorithm consists of three steps namely (i) Computation of neuronal signals (ii) Computation of error gradients and (iii) Weight updates [11].

a. Computation of neuronal signals: 
1. For the input layer:

$$
\begin{gathered}
\partial\left(x_{i}{ }^{k}\right)=x_{i}{ }^{k} \\
\partial\left(x_{o}{ }^{k}\right)=x_{o}{ }^{k}=1
\end{gathered}
$$

where $x_{i}{ }^{k}$ is the $i^{t h}$ component of the input vector presented the vector and $\partial\left(x_{o}{ }^{k}\right)$ is the input layer bias neuron signal that is independent of iteration index.

2. For the hidden layer:

$$
z_{h}{ }^{k}=\sum_{i=0}^{n} w_{i h}{ }^{k} \partial\left(x_{i}{ }^{k}\right)=\sum_{i=0}^{n} w_{i h}{ }^{k} x_{i}{ }^{k} \quad \mathrm{~h}=1,2 \ldots \ldots \mathrm{q}
$$

$$
\begin{array}{cr}
\partial\left(z_{h}{ }^{k}\right)=\frac{1}{1+e^{-z_{h}{ }^{k}}} & , \\
\partial\left(z_{0}{ }^{k}\right)=1 & \text { for all } \mathrm{k}=1,2 \ldots
\end{array}
$$$$
\mathrm{h}=1,2 \ldots . \mathrm{q}(33)
$$

where $w_{o h}{ }^{k}$ are the biases of the hidden neurons, and $\partial\left(z_{o}{ }^{k}\right)$ is the hidden layer bias neuron signal which is once again independent of iteration index.

\section{For the output layer:}

$$
\begin{gathered}
y_{j}{ }^{k}=\sum_{h=0}^{q} w_{h j}{ }^{k} \partial\left(z_{h}{ }^{k}\right) \quad \mathrm{j}=1,2 \ldots \ldots \ldots \ldots . . \mathrm{p} \\
\partial\left(y_{j}{ }^{k}\right)=\frac{1}{1+e^{-y_{j}{ }^{k}}} \quad, \quad \mathrm{j}=1,2 \ldots \ldots \ldots \ldots \mathrm{p}
\end{gathered}
$$

where $w_{o j}{ }^{k}$ are the biases of the output neurons.

\section{a. Computation of error gradient:}

\section{Hidden to output layer weights gradient:}

The error gradient $\frac{\partial \varepsilon}{\partial w_{h j}{ }^{k}}$ is given by the following equation

$$
\frac{\partial \varepsilon_{k}}{\partial w_{h j}{ }^{k}}=-\partial_{j}{ }^{k} \partial\left(z_{h}{ }^{k}\right)
$$

2. Input to hidden layer weight gradient:

$$
\frac{\partial \varepsilon_{k}}{\partial w_{i h}{ }^{k}}=-\partial_{h}{ }^{k} x_{i}{ }^{k}
$$

\section{b. Weight updates:} manner.

The weights are then updated in the following

1. For hidden to output layer weights:

$$
w_{h j}{ }^{k+1}=w_{h j}{ }^{k}+\eta \partial_{j}{ }^{k} \partial\left(z_{h}{ }^{k}\right)
$$

For input to hidden layer weights:

$$
w_{i h}{ }^{k+1}=w_{i h}{ }^{k}+\eta \partial_{h}{ }^{k} \partial\left(x_{i}{ }^{k}\right)
$$

The structure of the ANFIS that was developed after training the network is shown in the figure 7.

\section{Result}

The actual image was first enhanced in order to enable the proper extraction of the features. The segmented image which separates the background from the foreground area is shown in the Fig 8(a). The normalized image where the dynamic intensities are standardized so that the values are in a specific range is shown in Fig 8(b). The enhanced image is shown in the Fig 9(a) and this is better in terms of the continuity of the ridge structure and the Fig 9(b) shows the binarized image which has a better clarity and continuity of the ridge. This binarized image has only two levels of intensity values namely zero and one and clearly distinguishes between the ridges and the valleys.

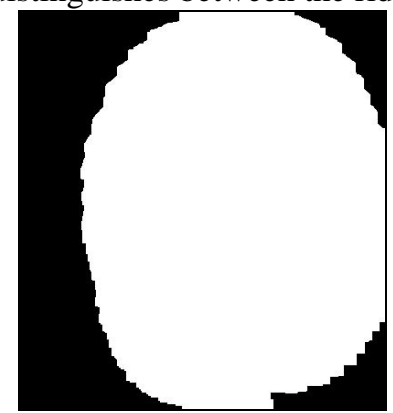

Fig 8. (a) Segmented image

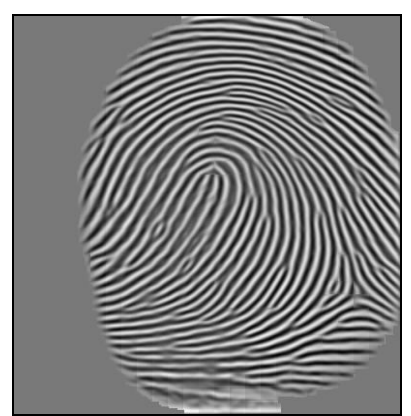

Fig 9. (a) Enhanced image

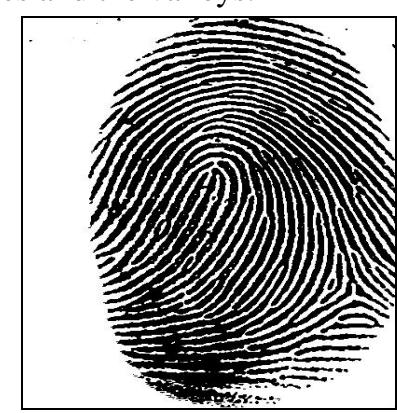

(b) Normalized image

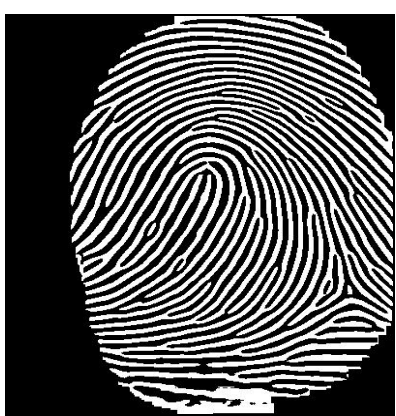

(b) Binarized image
The adaptive network when trained on the parameters proposed in this paper was found to be capable of correctly classifying the image into the proper category of ridge structure.

TABLE I: USING OUR METHODOLOGY

\begin{tabular}{|l|l|l|l|l|l|}
\hline $\begin{array}{l}\text { True class/Assigned } \\
\text { class }\end{array}$ & $\mathrm{R}$ & $\mathrm{L}$ & $\mathrm{W}$ & $\mathrm{A}$ & $\mathrm{T}$ \\
\hline $\mathrm{R}$ & 323 & 3 & 2 & 0 & 0 \\
\hline $\mathrm{L}$ & 4 & 341 & 1 & 0 & 0 \\
\hline $\mathrm{W}$ & 4 & 2 & 321 & 0 & 3 \\
\hline $\mathrm{A}$ & 0 & 0 & 2 & 206 & 0 \\
\hline $\mathrm{T}$ & 1 & 0 & 3 & 0 & 178 \\
\hline
\end{tabular}

TABLE II : USING TAN’ ET AL [12] METHODOLOGY

\begin{tabular}{|l|l|l|l|l|l|}
\hline $\begin{array}{l}\text { True class/Assigned } \\
\text { class }\end{array}$ & R & L & W & A & T \\
\hline $\mathrm{R}$ & 356 & 12 & 4 & 8 & 23 \\
\hline
\end{tabular}




\begin{tabular}{|l|c|c|c|c|c|}
\hline $\mathrm{L}$ & 4 & 372 & 6 & 9 & 3 \\
\hline $\mathrm{W}$ & 16 & 15 & 369 & 0 & 1 \\
\hline $\mathrm{A}$ & 3 & 3 & 0 & 416 & 8 \\
\hline $\mathrm{T}$ & 10 & 24 & 2 & 19 & 337 \\
\hline
\end{tabular}

The algorithm was tested on a database of 250 fingerprints. These were from a set of fifty different people, each contributing five samples. The test was carried out with the fingerprints from these fifty people whose template was stored in the database and fifteen others whose templates were not available. The test results with each of them testing five times are as follows:

Table III : Result using Proposed Algorithm with image ENHANCEMENT

\begin{tabular}{|c|c|c|}
\hline $\begin{array}{c}\text { Recognition } \\
\text { rate }\end{array}$ & $\begin{array}{c}\text { False Acceptance } \\
\text { (FRR) }\end{array}$ & $\begin{array}{c}\text { False Rejection } \\
\text { (FRR) }\end{array}$ \\
\hline $97.67 \%$ & $1.30 \%$ & $1.92 \%$ \\
\hline
\end{tabular}

TABle IV: Result using proposed algorl thM WI thout I Mage enhancement

\begin{tabular}{|c|c|c|}
\hline $\begin{array}{c}\text { Recognition } \\
\text { rate }\end{array}$ & $\begin{array}{c}\text { False Acceptance } \\
\text { (FRR) }\end{array}$ & $\begin{array}{c}\text { False Rejection } \\
\text { (FRR) }\end{array}$ \\
\hline $94.37 \%$ & $2.0 \%$ & $2.98 \%$ \\
\hline
\end{tabular}

TABLE V: RESULT USING G. AGUILAR' S [13] ALGORITHM

\begin{tabular}{|c|c|c|}
\hline $\begin{array}{c}\text { Recognition } \\
\text { rate }\end{array}$ & $\begin{array}{c}\text { False Acceptance } \\
\text { (FRR) }\end{array}$ & $\begin{array}{c}\text { False Rejection } \\
\text { (FRR) }\end{array}$ \\
\hline $94.1 \%$ & $2.3 \%$ & $3.6 \%$ \\
\hline
\end{tabular}

TABLE VI: RESULT USING ZHANG'S [14] ALGORITHM

\begin{tabular}{|c|c|c|}
\hline $\begin{array}{c}\text { Recognition } \\
\text { rate }\end{array}$ & $\begin{array}{c}\text { False Acceptance } \\
\text { (FRR) }\end{array}$ & $\begin{array}{c}\text { False Rejection } \\
\text { (FRR) }\end{array}$ \\
\hline $91.3 \%$ & $3.1 \%$ & $4.4 \%$ \\
\hline
\end{tabular}

The simulations were carried out on MATLAB 7.02 version on a $512 \mathrm{MB}$ RAM, $1.7 \mathrm{GHz}$ processor. The trained network was able to give the exact value every time an image which was one of those which had been trained earlier was given as input. The results were given fast and were observed to be correct for a large number of fingerprint inputs.

\section{CONCLUSION AND FUTURE WORK}

In this work, we have presented a new method wherein we use the image parameters like the mean, median, variance, standard deviation and root mean square value to describe an image. The images belonging to any particular group share a similarity in these parameters and hence make it possible for the adaptive network to learn the pattern and then classify the image accordingly. This method is a lot simpler than the previous techniques, wherein the extraction of the features from the image for the classification purposes was in itself an extremely tedious and time-consuming process. As the matching of the image also remains to be done, it is important to ensure that the classification of image is completed quickly.

Future work can be carried out in extending these parameter types in the matching of the fingerprint images. This involves spotting similar parameters which are very unique to a particular image and then using them in matching the images. This method of matching if found to be successful would make the entire matching technique simple and tremendously fast compared to the existing techniques.

\section{REFERENCES}

[1] P. M. K. Alobaidi, Fingerprint Image Enhancement, University Technology, Malaysia thesis, 1998.

[2] B. Meltem, F. A. Sakarya and B. L. Evans " A Fingerprint classification technique using directional images", IEEE Asilomar Conf. Signals, Systems and Computers, vol. 1, pp. 101-104, Nov 1997.

[3] L.C. Ern and G. Sulong "Fingerprint classification approaches- An overview", Proc of the sixth International Symposium on Signal processing and its applications, vol. 1, pp. 347-350, Aug 2001.

[4] G. T. Candela, P. J. Grother, C. I. Watson, R. A. Wilkinson and C. L. Wilson, " PCASYS- A pattern level classification automation system for fingerprints," NIST, Gaitherburg, MD, Tech. Rep. NISTIR 5647, Apr. 1995

[5] U. Halicini and G. Ongun, "Fingerprint classification through self-organizing feature maps modified to treat uncertainties", Proc. IEEE, vol. 84, no. 10, pp. 1497-1512, Oct.1996.

[6] A. K. Jain, S. Prabhakar and L. Hong, "A multichannel approach to fingerprint classification," IEEE Trans. Pattern Anal. Mach. Intell., vol. 21, no. 4, pp. 348-359, Apr.1999.

[7] Y. Yao, G. L. Marcialis, M. Pontil, P. Frasconi and F. Roli, "Combining flat and structured representations for fingerprint classification with recursive neural networks and support vector machines", Pattern Recog., vol. 36, no. 2, pp. 397-406, Feb 2003.

[8] A. Senior, "A combination fingerprint classifier", IEEE Trans. Pattern Anal. Mach. Intell., vol. 23, no. 10, pp. 1165-1174, Oct 2001.

[9] A. K. Jain and S. Minut, "Hierarchical kernel fitting for fingerprint classification and alignment", Proc. Int. Conf. Pattern Recog., vol. 2, pp. 469-473, Aug. 2002.

[10] J. Jang S R "ANFIS- Adaptive network based fuzzy inference system",IEEE Trans. On Systems, Man and Cybernetics, vol 23, no 3, June 1993.

[11] Satish Kumar, "Neural Networks", Tata McGraw-Hill Publications, 2004

[12] X. Tan, B. Bhanu and Y. Lin,"Fingerprint classification based on learned features",IEEE Trans on Systems, Man and Cybernetics, vol. 35, no.3, Aug 2005

[13] G. Aguilar, G. Sanchez, K. Toscano, M. Salinas, M. Nakano and H. Perez, "Fingerprint Recognition", Second International Conference on Internet Monitoring and Protection, pp. 32-32, July 2007.

[14] Y. Wang and W. Zhang, "Core Based Structure matching Algorithm of Fingerprint Verification", Proc. IEEE Intern. Conf. on Pattern recognition, Page 70-74,2002.

Rajesh Kumar received his $\mathrm{PhD}$ in Intelligent Systems from University of Rajasthan, India in 2005. He is currently working as associate professor at the Department of Electrical Engineering, Malaviya National Institute of Technology, Jaipur. He has authored more than 120 research papers in the area of intelligent systems, evolutionary algorithms, bio-inspired algorithms, intelligent motion control, image processing, power electronics, electrical machines and drives. He received the Career Award for Young Teachers in 2002. Dr. Kumar is a member of IEEE, IE (India), Fellow IETE, Senior member IAENG, member IEANG and Life Member of ISTE.

B R Deva Vikram was a Research Student at the Department of Electrical Engineering, Malaviya National Institute of Technology, Jaipur. He is now a graduate student in the Department of Electrical Engineering in Texas A\&M University USA. His areas of interests include artificial intelligence and image processing implementation. 